\title{
Improving 7-day working: face-to-face handover
}

\author{
Authors: William Rook, Wasim Mir, Jack Nash and Sarbjit Clare
}

\section{Aims}

To create a standardised efficient handover to improve patient safety and workflow for weekend on-call teams.

\section{Methods}

A weekly face-to-face handover meeting was initiated on Friday afternoon in the acute medical unit of an inner city hospital in Birmingham. Data were collected weekly from January until July, with a comparison made following recommendations. Those attending included the weekend ward-cover team: registrar, senior house officer, house officer, acute nurse practitioner, as well as representatives from the medical wards. The meeting was chaired by an acute medicine registrar or consultant. Matters discussed included discharge planning, necessity of investigations and ceilings of care. Attendants were encouraged to use the Royal College of Physicians (RCP) handover template.

\section{Results}

Initially, $38 \%$ of wards handing over jobs had a consultant ward round on a Friday, which improved to $55 \%$ at the end of the pilot. Use of the RCP handover template also improved from $40 \%$ to $96 \%$. Attendance remained variable, with $63-72 \%$ of wards attending. Many tasks were vetted and rejected by the seniors present, including unnecessary monitoring of blood tests and CT scans.

\section{Conclusions}

These data demonstrate how a standardised handover process, face to face, helps ensure a safe weekend for the on-call team. The meeting was an effective method to enable staff to discuss patients to be reviewed over the weekend, clarify plans, as well as to vet and filter any unnecessary jobs, helping ensure an appropriate workload.

Throughout this project we delivered a message to junior and senior doctors at a large hospital of how to optimise the current pattern for out-of-hours work with a brief and practical weekend handover. This ensured that the medical team had an effective way of managing continuity of care over a weekend to ensure optimum patient safety and patient flow. The most striking change achieved was the increase in a consultant presence at ward rounds on Fridays and in requesting investigations.

Many recent discussions regarding reconfiguration and optimisation of the NHS have focused on delivering a high standard of clinical care 7 days a week. Future strategies of this project will focus on improving attendance of all wards, through bleep reminds and prompts, as well as continued monitoring. The evidence from this project suggests that faceto-face communication is key in building teams and improving quality of care 7 days a week. 